\title{
Comparison of the Physical and Biomotor Characteristics, and Reaction Time between Turkish Male and Female Ice Hockey Players
}

\author{
Recep Gursoy ${ }^{1}$, Eser Aggon ${ }^{2}$, Robert Stephens ${ }^{3}$, Mehmet Akif Ziyagil ${ }^{4}$ \\ ${ }^{1}$ The Department of Physical Education and Sports, Atatürk University, Erzurum, Turkey \\ ${ }^{2}$ The Department of Physical Education and Sports, Erzincan University, Erzincan, Turkey \\ ${ }^{3}$ The Department of Physiology and Cell Biology, College of Medicine, The Ohio State University, \\ Columbus, USA \\ ${ }^{4}$ The Department of Physical Education and Sports, Amasya University, Amasya, Turkey \\ Email: recepgursoy@hotmail.com, eseraggon@gmail.com,drrobert.stephens@gmail.com, \\ Mehmet.ziyagil@gmail.com
}

Received July 19 ${ }^{\text {th }}$, 2012; revised August 25 $5^{\text {th }}$, 2012; accepted September $5^{\text {th }}, 2012$

\begin{abstract}
The purpose of this study was to measure and compare the physical and bio motor characteristics of male and female Turkish ice hockey players. Structural and functional differences depending on the gender were investigated. Altogether, 17 male and 20 female athletes from the city of Erzurum voluntarily participated in the study. The physical measurements of each player were recorded and then isometric strength, reaction time and flexibility were measured. Statistically, t-test analysis was independently used to compare the two groups. The results of the study showed the average weight and height of male athletes were higher than those of females, despite the fact that was lower. The average isometric leg, back and handgrip strength of male athletes were also significantly higher than those of females $(p<0.01)$. In the sit and reach test, females were better than those males $(p<0.01)$, while average body flexibility measurements of males are statistically higher than those of females $(p<0.05)$. There was no significant difference between two groups in vertical jump measurements. The average anaerobic capacity of males was significantly higher than those of females $(p<0.01)$. However, there was no significant difference in the average hand reaction time between two groups. Regular measurement of physical and functional characteristics of athletes is important not only in the talent identification of athletes but also in the understanding the gender related structural and functional differences as well as in the regulation of training programs.
\end{abstract}

Keywords: Sports Performance; Ice Hockey; Physical Characteristics

\section{Introduction}

Although female and male have played hockey for many years, a paucity of research data exist regarding the differential characteristics of male and female hockey players (Sapega \& Nicholas, 1982). Ice hockey is a sport that requires significant metabolic, physiological, and biomechanical skill (Ransdell \& Murray, 2011). It is a highly variable, high-intensity sport that requires speed, agility, muscular strength and endurance, and aerobic and anaerobic fitness (Burr et al., 2008; Noonan, 2010). Ice hockey has been depicted as a game played with hockey sticks, skates and pucks (Sim \& Chao, 1978; Mcfaull, 2001). Skating in ice hockey is a complex motor skill (Green, 2003) and it is uniquely stressful the high level of coordination required, the repeated demands made on the muscles with little rest and the astounding requirement that it is played while balancing on skate blades are all factors leading to rapid fatigue (Hansen \& Reed, 1979). In addition, ice hockey presents complex physical, physiological, and biomechanical challenges for players (Bracko et al., 1998; Marino, 1979), many experts believe the most important skill in ice hockey is skating (Hansen \& Reed, 1979). Several researchers have concluded that the 40-yd sprint (Bracko \& George, 2001), vertical jump (Mascaro, et al., 1992), and standing long jump (Burr et al., 2008) are valid and reliable predictors of on-ice test performance. To examine whether off-ice tests could predict game play (vs. on-ice skating performance), Green (2003) tested the relationship between player game performance (total minutes played and net scoring chances) and physical test results ( $V_{-}$O2max, blood lactate, and percent body fat). Understanding the physical performance characteristics of male and female hockey players can help identify weakness in conditioning, improve performance, establish baseline performance data and develop scientifically based training protocols. The game-performance skating characteristic, physical and biomotor characteristics, reaction time of ice hockey players are important for the team practitioners and coaches, because there are implications for on- and off-ice fitness training. Specifically, after Universiade Winter Games 2011 in Erzurum/Turkey women's and males participation in ice hockey and the other winter sports has grown about $\% 400$-fold during the past 2 years. Although several researchers studied physical and physiological profiles of ice hockey players, to our knowledge, there is no study, which has specifically addressed the characteristics of Turkish ice hockey players' physical and biomotor characteristics and, reaction time in the literature. This study is the first to report descriptive data from a sample of female ice hockey players from Turkey. 
Therefore this study aims to measure and compare the physical and biomotor characteristics, reaction time of male and female Turkish ice hockey players aged 17 to 25 years.

\section{Method}

37 healthy subjects comprising 17 male and 20 female ice hockey players from Erzurum region have voluntarily participated in this study. Height and body weight with short and t-shirt out were measured in the morning before breakfast. Height in centimeters and body weight in kilograms were recorded. Takei Kiki Kogyo Dynamometers were used to measure muscle strength between 1 and $100 \mathrm{~kg}$. For warm up, participants exercised 15 minutes prior to these measurements. A handgrip dynamometer was used to measure the isometric electronic hand strength, and an electronic leg dynamometer was used to measure the isometric leg and back strength. Reaction time was assessed by a software package of random stimulus presentation and response recording (Dane \& Erzurumluoglu, 2003). A monitor was placed $50 \mathrm{~cm}$ from each participant's eyes. A central fixation point on the screen was presented after a participant placed the chin in chin rest and aligned the eyes with the fixation point. To measure reaction time with right and left hands, a central flash as a visual stimulus was presented in response to which the participant was asked to press a key (A) on a keyboard. The participant's finger tip was about $1 \mathrm{~cm}$ from the key. Reaction times shorter than $150 \mathrm{msec}$. and longer than 500 msec. were removed from the analyses. Vertical jump was measured using the protocol of Baumgartner and Jackson (1987) with the average of the highest two of three jumps being recorded, anaerobic power was assessed using Sargent Jump Test. The hamstring and low back flexibility was determined by the sit-and-reach test. The athlete placed both feet (without shoes) flat against the Flex meter. The athlete reached as far forward as possible in a controlled manner while keeping the knees straight and palms facing down. The farthest distance reached was recorded to the nearest centimeter.

\section{Results}

T-test analysis independently determined differences between the physical characteristics and motor activity of male and female ice hockey players. The data testing are presented in Table $\mathbf{1 .}$

There was no difference between male and female ice hockey players in the mean age, training experience, left and right hand reaction time. Though the males had higher vertical jump scores than females, but difference was not significant between two groups. Male players had significantly higher mean height value than females $(p<0.05)$. The males had also significantly higher mean values than females in the following variables; body weight, isometric leg and back strength, right and left handgrip strength, and anaerobic power $(p<0.01)$. However, female players had significantly higher scores than males in sit-and-reach test $(p<0.01)$. The male players were the best in all tests except sit and reach test.

\section{Discussion}

The best of our knowledge, this study is the first to describe anthropometric and fitness performance characteristics of male and female ice hockey players from the Turkey. This study indicates that there were important structural and functional
Table 1.

Comparison of the physical and bio motor characteristics and, reaction time of male and female ice hockey players.

\begin{tabular}{ccc}
\hline Variable & Male (N = 17) & Female (N = 20) \\
\hline Age (years) & $18.71 \pm 1.57$ & $20.40 \pm 1.99$ \\
Training experience (years) & $1.82 \pm 0.81$ & $1.20 \pm 0.56$ \\
Weight $(\mathrm{kg})$ & $66.76 \pm 11.28^{* *}$ & $53.40 \pm 4.31$ \\
Height $(\mathrm{cm})$ & $173.12 \pm 7.44^{*}$ & $164.60 \pm 6.24$ \\
Isometric leg strength (kg) & $71.76 \pm 35.20^{* *}$ & $29.67 \pm 19.29$ \\
Isometric back strength (kg) & $70.71 \pm 28.04^{* *}$ & $25.40 \pm 13.69$ \\
Right handgrip strength (kg) & $43.12 \pm 6.60^{* *}$ & $28.73 \pm 4.74$ \\
Left handgrip strength (kg) & $43.47 \pm 7.05^{* *}$ & $26.33 \pm 4.59$ \\
Sit-and-reach (cm) & $8.41 \pm 5.40$ & $16.67 \pm 5.30^{* * *}$ \\
Vertical jump (cm) & $49.76 \pm 8.37$ & $41.73 \pm 7.23$ \\
$\begin{array}{c}\text { Anaerobic power }(\mathrm{kg} / \mathrm{m} / \mathrm{s}) \\
\text { Left hand reaction time } \\
(1 / 1000 \mathrm{~s})\end{array}$ & $104.25 \pm 21.20^{* *}$ & $75.97 \pm 7.50$ \\
$\begin{array}{c}\text { Right hand reaction time } \\
(1 / 1000 \mathrm{~s})\end{array}$ & $317.65 \pm 39.99$ & $314.80 \pm 31.97$ \\
\hline
\end{tabular}

Values are means and \pm is standard deviation of the means; ${ }^{*}$ Significant difference at $p<0.05$ levels; ${ }^{* *}$ Significant difference at $p<0.01$ levels.

differences between male and female ice hockey players. Males were heavier, taller, and powerful than females. Females were more flexible than males. Reaction time is accepted as an important measure of performance showing efficacy of speed, sensation and decision making, detection in sports activities (Kabakci, 2009). In this study there were no significant differences between male and female in the mean values of left hand, right hand, reaction time and vertical jump scores. Ransdell \& Murray (2011) did a study about physical profile of elite women ice hockey team from USA. In findings of them: 1) elite female ice hockey athletes were heavier, yet leaner than previously studied female athletes; 2) vertical jump was within the range previously reported for female collegiate Division I athletes; 3) standing long jump was slightly below the value previously reported for female collegiate Division I athletes; and 4) upper body and lower body strength (bench press and front squat, kilograms, respectively) were higher than the values previously reported for female collegiate Division I athletes. Vescovi et al. (2006) examined the physical and physiological characteristics of elite male ice hockey players with a mean age of $18.0 \pm 0.6$ years according to their playing positions. They concluded that there were significant differences in the view of anthropometric measurements, upper body strength, and anaerobic power among positions for elite-level ice hockey players Bracko et al., (2001) found that 12 - 13 years old male ice hockey players had higher mean values of body weight. Also 14 - 15 years old males were more powerful than females. These results were parallel with findings of our study. But our flexibility results were not consistent with findings his because there was no difference in the flexibility of the players in study of Bracko (2001). In the study of Bracko et al. (2001) all groups had similar acceleration performance. Similarly, Bracko (2001) 
found insignificant difference in the accelerating ability between elite and non-elite female ice hockey players. Bracko et al., (1998) also found that non-age matched male high school players were similar in acceleration, Bracko and Fellingham (2001) reported that it appears that the accelerating ability did not differentiated significantly for younger and older elite and non-elite females, and young non-elite male hockey players. But male ice hockey players were faster than the females on the speed test. Dane and Erzurumluoglu (2003) reported that the eye-hand reaction time was of longer duration for women than men. But we didn't find difference between male and female ice hockey players training experience, left and right hand reaction time in this study. According to Montegomerty (1998) anaerobic fitness is an important performance variable for hockey players, and information about on-ice fitness can be an important criteria for a coach to understand a player's ability. Bar-Or (1987) suggests the accuracy of "field tests" maybe questioned because of the skill level required to perform well. Nevertheless, skating ability, and testing skating ability are important aspects of hockey performance (Hansen \& Reed, 1979). Bracko (2001) found that 10 - 11, 12 - 13, 14 - 15 years male ice hockey players have higher anaerobic power than female. Sport scientists are concerned with eliminating the skill factor in exercise testing to produce objective results, whereas coaches are interested in a player's sport-specific fitness and game-performance skating ability (Watson \& Sargeant, 1986). Future research is needed to examine the relationship between training and fitness levels, to compare on-ice performance tests with off-ice fitness test results, and to continue to examine potential positional differences in fitness parameters in male and female ice hockey players.

As a result, knowing the physical profile of male and female ice hockey players can enable athletes, coaches, athletic trainers, scouts, and strength and conditioning specialists to establish athlete physical fitness expectations, design science-based training programs that will improve performance, and address any weaknesses in physical fitness identified through testing. Also the current findings can be used to identify physical characteristics and biomotor abilities for male and female ice hockey players (average for man $1.82 \pm 0.81$, for women $1.20 \pm 0.56$ ) and regular measurement of physical and functional characteristics of athletes is an important method used in comparing athletes from two different teams as well as in evaluating the efficiency of their training programs.

\section{REFERENCES}

Bar-Or, O. (1987). The Wingate anaerobic test: An update on methodology, reliability and validity. Sports Medicine, 4, 381-394.

Baumgartner, T., \& Jackson, A. S. (1987). Measurement for evaluation in physical education and exercise science. Dubuque, IA: W. M. C. Brown. doi:10.1097/00005768-199605001-00324

Bracko, M. R., Hall, L. T., Fisher, A. G., Fellingham, G. W., \& Cryer, W. (1998). Performance skating characteristics of professional ice hockey forwards. Sports Medicine Training and Rehabilitation, 8, 251-263. doi:10.1080/15438629809512531

Bracko, M. R. (2001). On performance characteristics of elite and non-elite female ice hockey players. The Journal of Strength \& Conditioning Research, 1, 42-47.

Bracko, M. R., \& Fellingham, G. W. (2001). Comparison of physical performance characteristics of female and male ice hockey players. Pediatric Exercise Science, 13, 26-34.

Bracko, M. R., \& George, J. D. (2001). Prediction of ice skating performance with off-ice in women's ice hockey players. The Journal of Strength \& Conditioning Research, 15, 116-122.

Burr, J. F., Jamnik, R. K., Baker, J., Macpherson, A., Gledhill, N., \& Mcguire, E. J. (2008). Relationship of physical fitness test results and hockey playing potential in elite-level ice hockey players. The Journal of Strength \& Conditioning Research, 22, 1535-1543. doi:10.1519/JSC.0b013e318181ac20

Dane, S., \& Erzurumluoglu, A. (2003). Sex and handedness differences in eye-hand visual reaction times in handball players. International Journal of Neuroscience, 113, 923-929.

doi:10.1080/00207450390220367

Green, H. (2003). Personal communication, Janaury 17 by Michael R. Bracko.

Hansen, H., \& Reed, A. (1979). Functions and on-ice competencies of a high caliber hockey player-A job analysis. In J. Terauds, \& H. J. Gros (Eds.), Science in skiing, skating, and hockey (pp. 107-115). Del Mar, CA: Academic Publishers.

Kabakci, A. C. (2009). The differences in reaction times of elite football, handball and ice hockey goal keepers. Ph.D. Thesis, Ankara: Gazi University.

Marino, G. W. (1979). Acceleration-time relationships in an ice skating start. Research Quarterly for Exercise \& Sport, 50, 55-59.

Mascaro, T., Seaver, B. L., \& Swanson, L. (1992). Prediction of skating speed with off-ice testing in professional hockey players. Journal of Orthopedic \& Sports Physical Therapy, 10, 92-98.

Mcfaull, S. (2001). Contact injuries in minor hockey: A review of the Chirpp database for the 1998/1999 hockey season. The Canadian Hospitals Injury Reporting and Preventing Program News, 19, 1-9.

Montegomerty, D. L. (1998). Physiology of ice hockey. Sports Medicine, 5, 99-126. doi:10.2165/00007256-198805020-00003

Noonan, B. C. (2010). Intragame blood-lactate values during ice hockey and their relationships to commonly used hockey testing protocols. The Journal of Strength \& Conditioning Research, 24, 2290-2295. doi:10.1519/JSC.0b013e3181e99c4a

Sapega, A., \& Nicholas, J. A. (1982). Clinical use of musculoskeletal profiling in orthopedic sports medicine. The Physician and Sports Medicine, 9, 80-88.

Sim, F. H., \& Chao, E. Y. (1978). Injury potential in modern ice hockey. The American Journal of Sports Medicine, 6, 378-384. doi:10.1177/036354657800600612

Ransdell, L. B., \& Murray, T. A. (2011). Physical profile of elite female ice hockey players from the USA. Journal of Strength \& Conditioning Research, 25, 2358-2363. doi:10.1519/JSC.0b013e31822a5440

Vescovi, J. D., Murray, T. M., \& Van Heest, J. L. (2006). Positional performance profiling of elite ice hockey players. International Journal of Sports Physiology and Performance, 1, 84-94.

Watson, R. C., \& Sargeant, T. L. C. (1986). Laboratory and on-ice test comparisons of anaerobic power of ice hockey players. Canadian Journal of Applied Sciences, 11, 218-224. 